\title{
PacBio sequencing reveals bacterial community diversity in cheeses collected from different regions
}

\author{
Chengcong Yang, Feiyan Zhao, Qiangchuan Hou, Jiao Wang, Min Li, and Zhihong Sun* \\ Key Laboratory of Dairy Biotechnology and Engineering, Key Laboratory of Dairy Products Processing, Inner Mongolia Agricultural University, \\ Hohhot 010018, P. R. China
}

\begin{abstract}
Cheese is a fermented dairy product that is popular for its unique flavor and nutritional value. Recent studies have shown that microorganisms in cheese play an important role in the fermentation process and determine the quality of the cheese. We collected 12 cheese samples from different regions and studied the composition of their bacterial communities using PacBio small-molecule real-time sequencing (Pacific Biosciences, Menlo Park, CA). Our data revealed 144 bacterial genera (including Lactobacillus, Streptococcus, Lactococcus, and Staphylococcus) and 217 bacterial species (including Lactococcus lactis, Streptococcus thermophilus, Staphylococcus equorum, and Streptococcus uberis). We investigated the flavor quality of the cheese samples using an electronic nose system and we found differences in flavor-quality indices among samples from different regions. We found a clustering tendency based on flavor quality using principal component analysis. We found correlations between lactic acid bacteria and the flavor quality of the cheese samples. Biodegradation and metabolism of xenobiotics, and lipid-metabolismrelated pathways, were predicted to contribute to differences in cheese flavor using Phylogenetic Investigation of Communities by Reconstruction of Unobserved States (PICRUSt). This preliminary study explored the bacterial communities in cheeses collected from different regions and their potential genome functions from the perspective of flavor quality.
\end{abstract}

Key words: cheese, flavor quality, PacBio SMRT sequencing, bacterial diversity

\section{INTRODUCTION}

Cheese is a fermented dairy product rich in nutrients such as proteins, amino acids, fats, and inorganic salts (Salameh et al., 2016). It has a large consumer mar-

Received August 26, 2019.

Accepted October 28, 2019.

*Corresponding author: sunzhihong78@163.com ket, both in China and globally. With the development of modern food science technologies, the cheese production process has become industrialized. Although modern technology has greatly improved the efficiency of cheese production, microbial communities in cheese have undergone great change (Yeluri Jonnala et al., 2018). Because of substantial differences in cultures, environments, and customs among regions, cheese production methods also differ widely. Dugat-Bony et al. (2016) observed patterns specific to the cheese production facility, underlining the importance of indigenous microorganisms for the assemblage of cheese microbiota. Samelis and Kakouri (2019) evaluated industrial Galotyri cheese technology in a fresh yogurt-like cheese with limited lactic acid bacteria (LAB) diversity and found that the artisan-type Galotyri cheese technologies varied significantly but shared a decisive ripening step that favored the predominance of mesophilic LAB species in the final products. In general, traditional cheeses are made by fermenting fresh milk in wooden or porcelain barrels for a period of time. The fermented milk is then slowly heated until it thickens (Scatassa et al., 2015). To improve the taste, butter and cream are usually added before shaping and ripening (Mania et al., 2018). The fermentation process and the ultimate quality of the cheese is affected by the microorganisms that are naturally present (Mania et al., 2018). These microorganisms come mainly from the production environment, the molding process, and the raw materials (Stellato et al., 2015; Licitra et al., 2018). In recent years, cheese-related research has focused largely on storage (Felicio et al., 2016), production processes (Blaiotta et al., 2017), and sensory properties (Koca et al., 2015). However, previous studies have reported that the microbes present in cheese might also be important for taste, flavor, and nutritional value (O'Sullivan et al., 2015). The exact role of bacteria in the quality of final cheese products remains inadequately studied.

As the most common microorganisms in cheese, LAB participate directly in the fermentation process and contribute greatly to a cheese's sensory and physical properties (Pedersen et al., 2016). Linares et al., (2017) 
found that $\mathrm{LAB}$ produce a wide range of bioactive compounds that remain in dairy products after milk fermentation. Some of these bioactive compounds have anti-inflammatory, anti-hypertensive, or anti-diabetic properties (Fujita et al., 2017; Georgalaki et al., 2017). As well, LAB influence the taste, aroma, and texture of fermented foods (Liu and Narbad, 2018). They produce a variety of extracellular and capsular polysaccharides that contribute to the characteristic textural properties of different types of cheeses (Caggianiello et al., 2016; Zeidan et al., 2017). Improving our understanding of the bacterial community associated with cheese, particularly the LAB, could help in the design of cheese products with desirable qualities and health-promoting functions.

High-throughput sequencing technology has been used widely in the study of microbial communities from environmental samples, including foods (Wang et al., 2018). PacBio small-molecule real-time (SMRT) sequencing technology (Pacific Biosciences, Menlo Park, $\mathrm{CA}$ ) has the ability to read long sequences that is unmatched by second-generation sequencing technology (Koren et al., 2012). The circular consensus sequencing strategy is based on repeated sequencing of each DNA strand and was developed to rectify sequencing errors in PacBio SMRT sequencing technology (Rhoads and $\mathrm{Au}$, 2015); full-length 16S rRNA gene profiling by PacBio sequencing can now be used to accurately describe the composition of the bacterial community in samples to the species level (Schloss et al., 2016; Wagner et al., 2016). This is a convenient way to describe the community structure and composition of bacteria in food samples, including fermented foods (Lima-Ojeda et al., 2017).

In this study, we used PacBio SMRT sequencing and electronic nose technology to analyze the bacterial diversity and flavor quality of traditional cheese samples collected from different regions, as well as to investigate the correlation between bacterial diversity and the flavor quality of cheese. Our findings could have reference significance for large-scale production enterprises. At the same time, defining the bacterial microbial information in traditional cheese, is helpful for protecting strain resources, developing high-quality industrial strains, and producing excellent cheese.

\section{MATERIALS AND METHODS}

\section{Sample Collection}

A total of 12 samples of traditional fermented cheese were collected from 12 regions from around the world. Globally, these regions are all recognized for the tradi- tional production of cheese. For full information on the samples, please refer to Supplemental Table S1 (https: //doi.org/10.3168/jds.2019-17496). Approximately 50 $\mathrm{g}$ from each sample was transferred into sealed sterile bags using a sterile spoon, and samples were labeled LFY1 to LFY12. Samples were sent to the Key Laboratory of Dairy Biotechnology and Engineering of Inner Mongolia Agricultural University (Hohhot, China) on dry ice and stored at $-80^{\circ} \mathrm{C}$ until evaluation.

\section{DNA Extraction}

Each cheese sample collected was thoroughly ground to powder, and then $0.5 \mathrm{~g}$ was used for experiments. We extracted macrogenomic DNA using the DNeasy PowerFood kit (Omega Bio-tek Inc., Norcross, GA) according to the manufacturer's instructions (https://www .omegabiotek.com). The main steps were as follows: (1) add $0.5 \mathrm{~g}$ of microbial food to a $2-\mathrm{mL}$ collection tube, resuspend the cell pellet in $450 \mu \mathrm{L}$ of solution MBL and vortex to mix; (2) centrifuge the tubes at a maximum of $13,000 \times g$ for $1 \mathrm{~min}$ and transfer the supernatant to a clean $2 \mathrm{~mL}$ collection tube; (3) add $100 \mu \mathrm{L}$ of solution IRS, vortex briefly to mix, and incubate at 2 to $8^{\circ} \mathrm{C}$ for $5 \mathrm{~min}$; (4) transfer $450 \mathrm{~mL}$ of supernatant to a clean $2 \mathrm{~mL}$ collection tube and add $900 \mu \mathrm{L}$ of solution $\mathrm{MR}$ and vortex to mix; (5) load $650 \mu \mathrm{L}$ of supernatant onto an MB spin column, centrifuge at $13,000 \times g$ for $1 \mathrm{~min}$, and place the MB spin column into a clean 2 $\mathrm{mL}$ collection tube; (6) add solution PW, ethanol, and solution EB successively; (7) after adding each reagent, centrifuge at $13,000 \times g$ for $1 \mathrm{~min}$ and discard the flowthrough; (8) collect DNA.

We evaluated the purity, concentration, and integrity of the extracted DNA using 1.0\% agarose gel electrophoresis and a micro-UV spectrophotometer (Nanodrop One, Thermo Fisher Scientific, Waltham, MA). All high-quality DNA was stored at $-20^{\circ} \mathrm{C}$ for analysis.

\section{Amplification of Full-Length 16S rRNA}

The full-length 16S rRNA genes were amplified using the $27 \mathrm{~F}$ and $1541 \mathrm{R}$ primer pair (27F: 5'-AGAGTTTGATCCTGGCTCAG-3'; 1495R: 5'-CTACGGCTACCTTGTTACGA-3'). Twelve nucleotide barcode labels were incorporated into each primer for sample labeling. Each PCR amplification reaction mix consisted of $4 \mu \mathrm{L}$ of $5 \times$ PCR buffer; $2 \mu \mathrm{L}$ of $2.5 \mathrm{mM}$ dNTP mix; $0.8 \mu \mathrm{L}$ of $5 \mu \mathrm{mol} / \mathrm{L}$ forward primer; $0.8 \mu \mathrm{L}$ of $5 \mu \mathrm{mol} / \mathrm{L}$ reverse primer; $0.4 \mu \mathrm{L}$ of $5 \mathrm{U} / \mu \mathrm{L}$ DNA polymerase; 10 ng of DNA template; and distilled deionized $\mathrm{H}_{2} \mathrm{O}$ added to achieve a final volume of $20 \mu \mathrm{L}$ (all reagents used in PCR amplification are from Kapa HiFi HotStart 
ReadyMix, Kapa Biosystems, Wilmington, MA). The PCR amplification conditions were as follows: $95^{\circ} \mathrm{C}$ for $3 \mathrm{~min} ; 30$ cycles of $95^{\circ} \mathrm{C}$ for $30 \mathrm{~s}, 55^{\circ} \mathrm{C}$ for $30 \mathrm{~s}, 72^{\circ} \mathrm{C}$ for $45 \mathrm{~s} ; 72^{\circ} \mathrm{C}$ for $10 \mathrm{~min}$.

\section{PacBio SMRT Sequencing and Quality Control}

The purified PCR products were subjected to library construction using the SMRT bell Template Prep Kit 1.0 kit (Pacific Biosciences). We used the Rs II instrument P6-C4 reagent (PacBio) for sequencing, according to the instructions provided by the manufacturer (https://www.pacb.com/wp-content/uploads/2015/ 09/Unsupported-Amplicon-Template-Preparation -Sequencing.pdf).

The quality of the original sequences generated was controlled using RS_ReadsOflnsert.1 (Hou et al., 2015). The criteria for high-quality full-length $16 \mathrm{~S}$ rRNA sequences were as follows: (1) the number of repeated sequencing of the insert was $\geq 5$; (2) the minimum prediction accuracy was $90 \%$; (3) the minimum insertion sequence length was 1,400 nt; and (4) the maximum sequence length was 1,800 nt. Sequences that met these criteria were evaluated further.

\section{Bioinformatic Analysis}

Sequences were assigned to different samples based on the barcodes. The samples were subjected to analysis and bacterial diversity evaluation using the QIIME platform (version 1.70; Caporaso et al., 2012). The specific bioinformatic analysis process was as follows: (1) calibration and alignment of high-quality sequences using PyNAST software (Caporaso et al., 2010); (2) construction of a matrix of operational taxonomic units (OTU) with UCLASS (Edgar, 2010) by first applying $100 \%$ similarity to establish a full-length sequence set of non-redundant $16 \mathrm{~S}$ rRNA genes, and then UCLASS partitioning under $97 \%$ similarity; (3) removal of chimeric sequences in the constructed OTU matrix using ChimeraSlayer software (version 1.7.0-dev, https:// omictools.com/chimeraslayer-tool); (4) taxonomic assignment using the Greengenes (version 13.8; DeSantis et al., 2006), RDP Release (release 11.5; Maidak et al., 2000), and SILVA databases (version 128; Quast et al., 2013); (5) construction of phylogenetic tree using FastTree software (Cao et al., 2017) based on the representative OTU sequence; (6) calculation of $\alpha$-diversity indices (number of observed species index, Shannon, Chao1, and Simpson indices) to assess microbial species richness and diversity in each sample; we plotted the Shannon index curve and the number of observed species to evaluate the requirements for subsequent bio- informatics analysis at the time of sequencing depth; (7) random leveling of the number of sequences from each sample based on samples with the smallest sequence number; we repeated this step 1,000 times to minimize the error caused by the leveling and combined the results of the 1,000-level flattening for $\beta$ diversity analysis; (8) prediction of the microflora function of bacteria in cheese using Phylogenetic Investigation of Communities by Reconstruction of Unobserved States (PICRUSt) software (Douglas et al., 2018) and annotation based on the Kyoto Encyclopedia of Genes and Genomes (KEGG) database (Kanehisa et al., 2017).

\section{Determination of Cheese Flavor Characteristics}

Five grams of each cheese samples was sealed in an electronic nose test bottle, heated in a water bath at $50^{\circ} \mathrm{C}$ for $10 \mathrm{~min}$, and stored at room temperature for 30 min. The electronic nose sensor (PEN3 Electronic Nose, Airsense Analytics GmbH, Schwerin, Germany) is composed of 10 kinds of metal oxide semiconductor (MOS) chemical sensing elements, and each type of sensing element differs by type of main sensitive substance. By running an improved desorption unit, which has the functions of automatic adjustment, automatic calibration, and automatic enrichment of the system, the conductivity of the sensor is changed using the interaction between the sensor and the gas. The change value of resistance in the circuit is used to output the signal. Cheese fermented by different bacterial strains have different metabolites and present different odor signals to the sensor. After $90 \mathrm{~s}$ of automatic cleaning of the metal electrode, the flavor of the sample was determined for $90 \mathrm{~s}$, and the electronic nose measured the response value per second. Here, response values of $69 \mathrm{~s}, 70 \mathrm{~s}$ and $71 \mathrm{~s}$ were selected as test data. Each sample was tested 3 times.

\section{Statistical Analysis}

The number of different types of OTUs in the samples was analyzed using the UpSetR package in $\mathrm{R}$ software (Conway et al., 2017). We conducted principal components analysis (PCA) using PAST Software (Hammer et al., 2001). We used Canoco software (version 4.50; Gilliam and Saunders, 2003) for redundancy analysis and to screen for differential metabolic pathways. Spearman rank correlation analysis and the Wilcoxon test were used to analyze co-occurrence patterns and differences between dominant bacteria (those with average relative abundance $>0.5 \%$ ) and flavor quality. Cytoscape software (version 3.5.1, Cytoscape Consortium; https://cytoscape.org/) was used to draw 
the correlation network diagram. Significant correlations among dominant species and metabolic pathways were visualized by heatmap. Redundancy analysis was used to identify the key indicators accounting for differences. We plotted graphic presentations using $\mathrm{R}$ (version 3.3.2; https://www.r-project.org/) and Origin 2017 (OriginLab Corp., Northampton, MA).

\section{Data Deposition}

All DNA sequences generated in this work have been uploaded to the Metagenomic Rapid Annotations using Subsystem Technology (MG-RAST) database under the project number mgp89891.

\section{RESULTS AND DISCUSSION}

\section{Species and Diversity Analysis}

A total of 40,562 high-quality $16 \mathrm{~S}$ rRNA genes sequences were obtained from across all samples, corresponding to an average read number for each sample of 3,380 (range $=2,095-4,276 ; \mathrm{SD}=655$ ). After $100 \%$ similarity cluster analysis, we obtained 18,050 representative reads. After UCLASS partitioning under $97 \%$ similarity, 1,459 OTU remained. We removed 88 chimeric OTU, leaving 1,371 OTU for further analysis.

We used $4 \alpha$-diversity indices to evaluate the species richness and evenness of samples (Figure 1; Supplemental Table S2, https://doi.org/10.3168/jds.2019-17496). The rarefaction curves for the number of observed species did not saturate as the Shannon index curves leveled off, suggesting that good representation of the bacterial diversity had been captured by the sequencing depth used, although new taxa could still be discovered by further sequencing.

At the phylum level, the cheese community from all samples consisted mainly of 8 bacterial phyla, dominated by Firmicutes and Actinobacteria, with average relative abundances of $88.25 \%$ and $7.23 \%$, respectively. We identified a total of 144 bacterial genera (Figure $2)$; the dominant genera with an average relative abundance $>1 \%$ were Lactobacillus (25.52\%), Streptococcus $(23.13 \%)$, Lactococcus $(17.66 \%)$, Staphylococcus $(12.75 \%)$, Leuconostoc $(7.53 \%)$, Brevibacterium $(5.22 \%)$, and Bartonella (1.60\%). We identified 218 bacterial species, of which 16 represented $>0.5 \%$ of all species present, and $89.47 \%$ of all sequences.

Previous studies have shown that LAB use fat hydrolases, protein hydrolases, and amino acid inverters to convert various milk components to a variety of volatile flavor compounds (Bourdichon et al., 2012). Lactobacillus and Streptococcus species are widely reported as the dominant bacteria in cheese (Aldrete-Tapia et al., 2014;
Treu et al., 2017). They are the most common fermentative bacteria in yogurt and contribute significantly to the flavor quality of cheese. Furthermore, Lactobacillus species use milk components to produce a variety of metabolites, including alcohols, aldehydes, esters, ketones, and acids, which give rise to the characteristic flavor of fermented milk products (Pogačić et al., 2016). Streptococcus species also contribute significantly to flavor formation in dairy products (Dan et al., 2017). Lactobacillus and Streptococcus species are often used together as starter cultures in the manufacture of fermented dairy products; their complementary activity
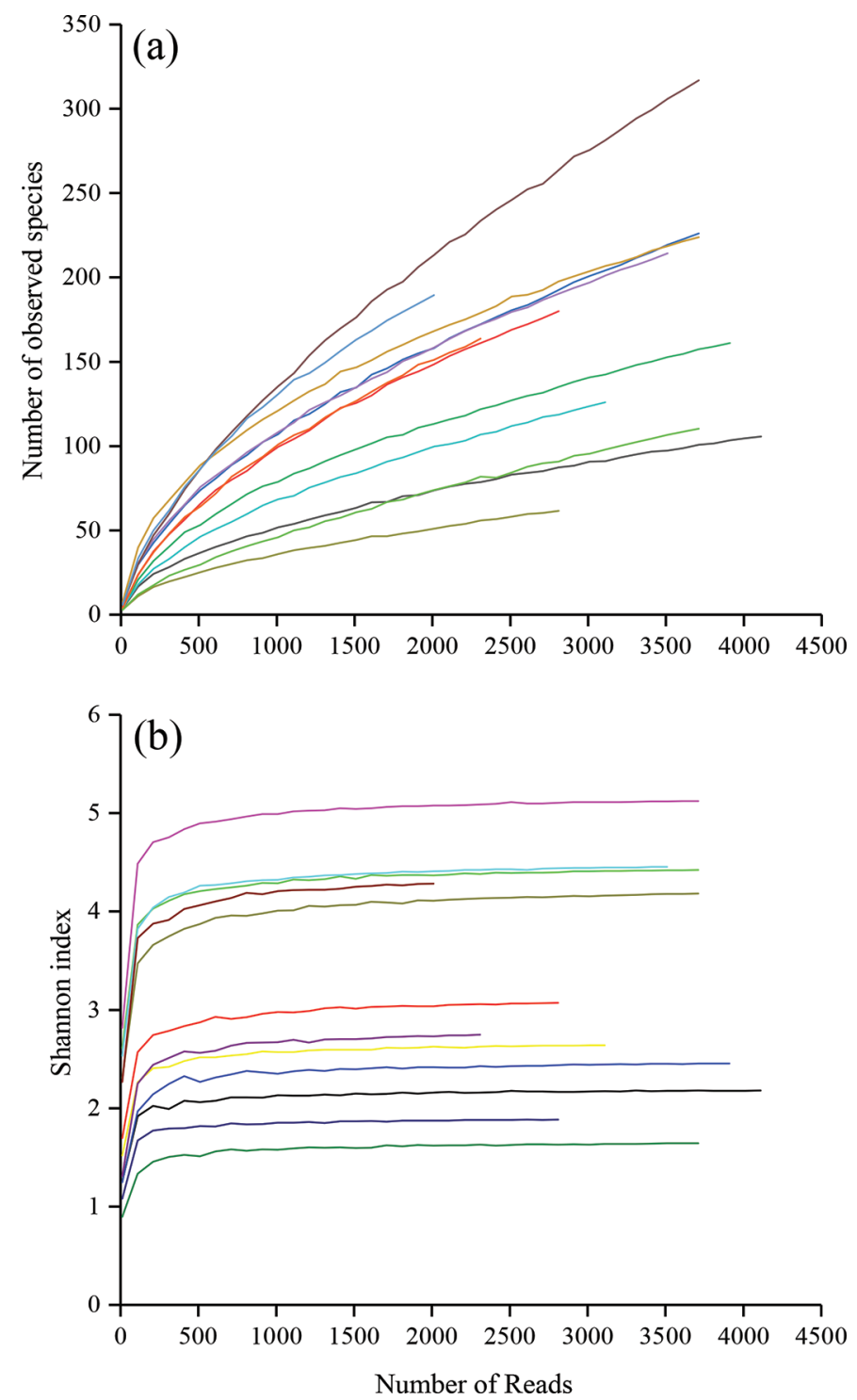

Figure 1. (a) Rarefaction, and (b) Shannon diversity curves of 12 cheese samples from different regions analyzed using PacBio smallmolecule real-time sequencing (Pacific Biosciences, Menlo Park, CA). 
endows fermented dairy products with special flavor qualities (Sørensen et al., 2016).

At the species level, we observed large variation between samples. For example, Lactococcus lactis was the dominant species in samples LFY8, LFY9, and LFY11, and Streptococcus thermophilus was the dominant species in samples LFY1 and LFY3. Large compositional differences in bacterial communities between samples with different geographical origins have been reported previously, attributed to different manufacturing environments, which can influence the dynamics and diversity of bacterial communities (Kamimura et al., 2019). Moreover, altitude (Zheng et al., 2018), air humidity (Stone et al., 2016), culture (Dugat-Bony et al., 2016), and manufacturing technology (Sádecká et al., 2016) are also potential influencing factors. It is worth noting that, although samples LFY6, LFY7, and LFY8 were all collected from Italy, their bacterial communities still had compositional differences. This suggests that cheese production processes could have a great effect on bacterial communities in cheese.

\section{Common OTU and Core OTU in Cheeses from Different Regions}

We also counted the common OTU (those that appeared in 2 or more cheese samples) and unique OTU (those that appeared in only 1 cheese sample) from different regions. None of the OTU were shared by all samples; only 13 OTU were shared by 3 samples, and 85 were shared by 2 samples (Figure 3 ).

Among the common OTU, 43 were not identified at the species level, accounting for $3.02 \%$ of all reads. The annotated results for common OTU showed that the highest proportions were in Lc. lactis (18.37\%), Strep. thermophilus (16.66\%), Streptococcus uberis (10.72\%),

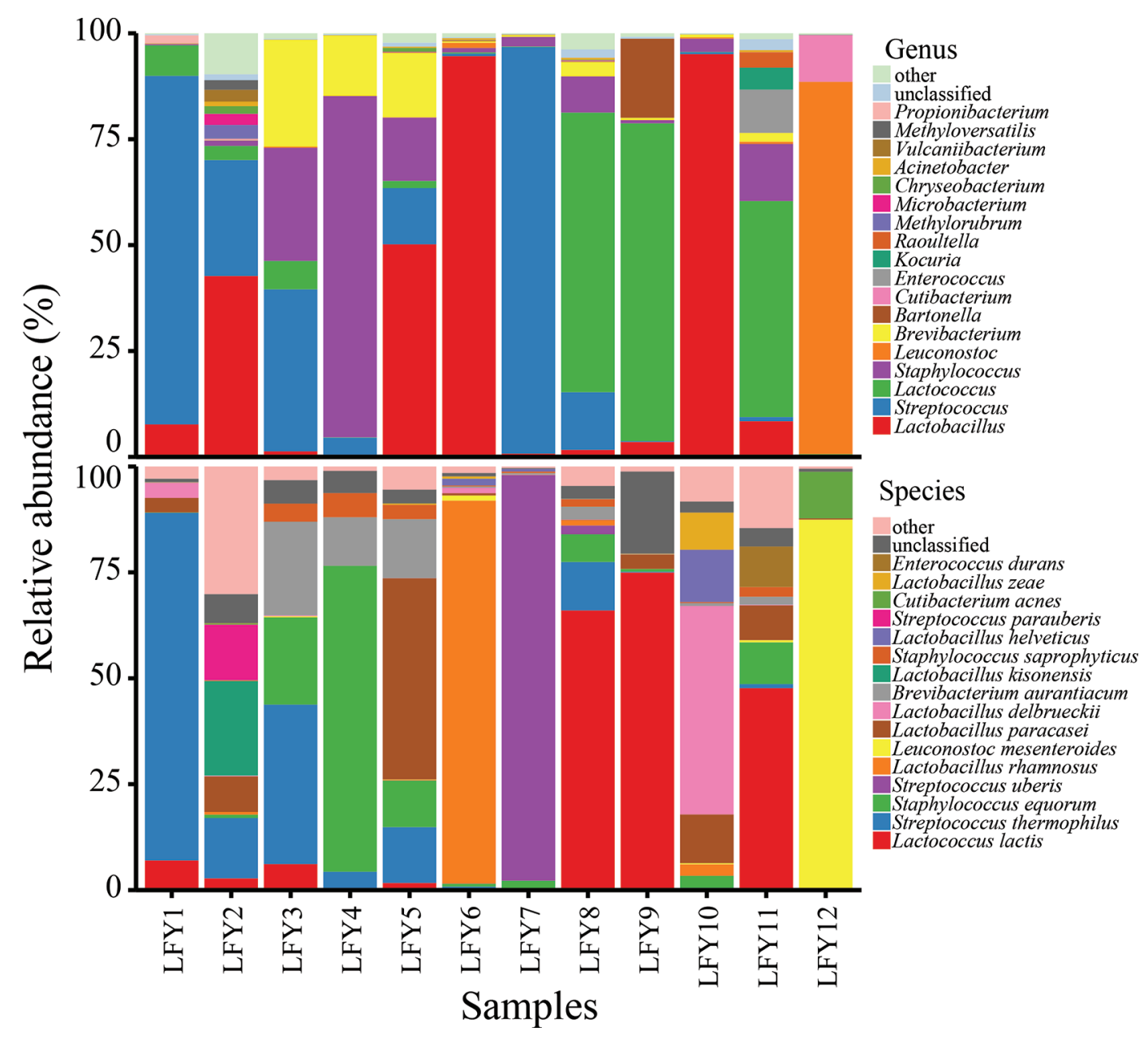

Figure 2. Relative abundances of the major bacterial genera and species in cheese samples from different regions analyzed using PacBio small-molecule real-time sequencing (Pacific Biosciences, Menlo Park, CA). LFY indicates sample numbers. 


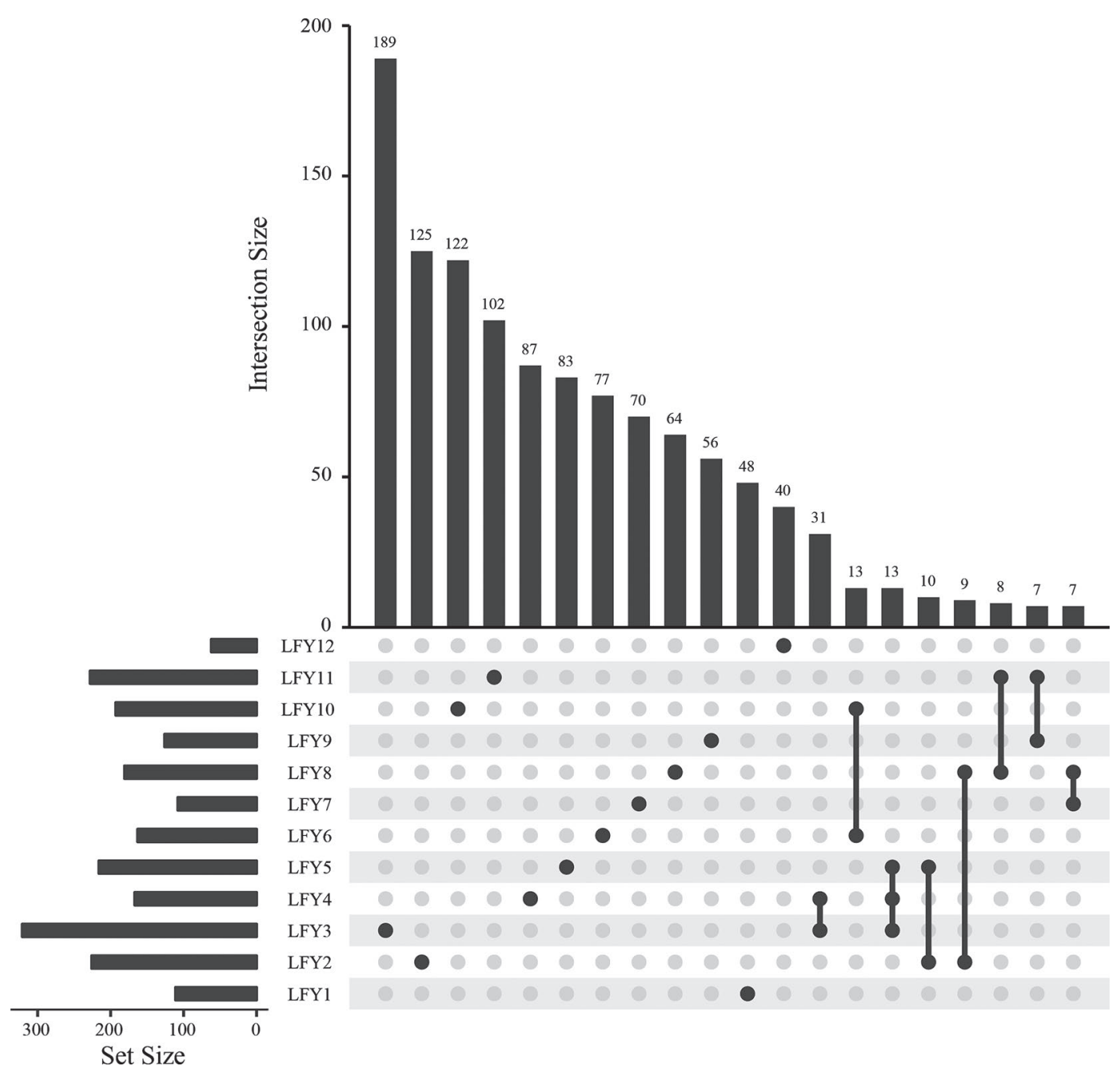

Figure 3. Bacterial shared operational taxonomic units (OTU) in cheese samples from different regions (only shared OTU numbering more than 7 are shown). LFY indicates sample numbers.

and Staphylococcus equorum (10.15\%). Samples LFY3, LFY2, LFY10, and LFY11 had a large number of unique OTU in addition to the common OTU: 189, 125,122 , and 102 , respectively. This shows that the bacteria from different cheese samples vary greatly.

To determine the overall difference in bacterial community structure among the different samples, we used PCA based on weighted and unweighted UniFrac distances (Supplemental Figure S1; https://doi.org/10 .3168/jds.2019-17496). In general, samples from different regions showed a particular separation trend on weighted and unweighted score maps, and samples from the same country showed no obvious clustering trend. Physical distance and different production methods had a great influence on bacterial community structures in cheese (Jin et al., 2018).

\section{Correlation Between Flavor Quality and Bacterial Communities in Cheese}

We evaluated the flavor quality of cheese using the electronic nose technique. The relative intensity of different flavor evaluation indices is shown in Table 1 (Hai and Wang, 2006).

The results of electronic nose analysis showed some differences in the relative intensity of flavor indices among the different cheese samples. The largest variation was recorded using the electrode W5S (56.81\%), which measures mainly nitrogen oxide content. This finding suggests that nitrogen oxide could be an important contributor to the flavor quality of cheese. We also found large variations between samples using electrodes W1S (27.30\%), W2S (28.01\%), W1W (41.84\%), 
Table 1. Flavor-quality indices of cheese samples determined by different electrodes

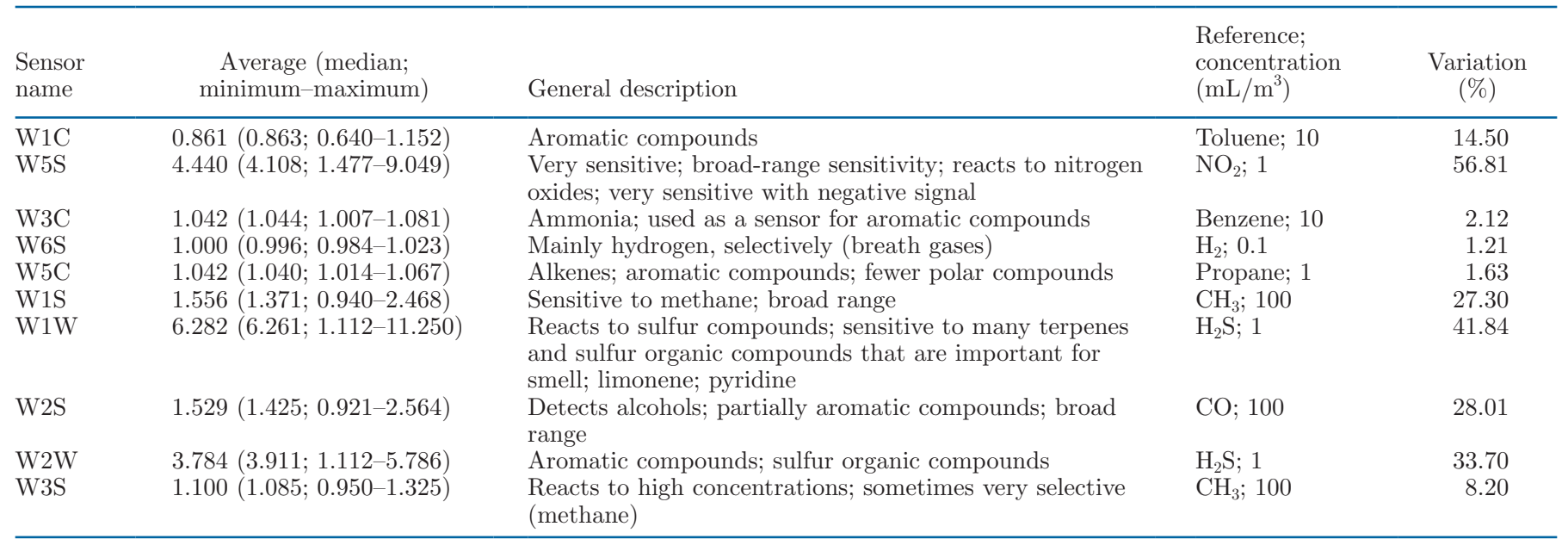

and W2W (33.70\%), respectively. We found relatively little variation using electrodes W1C $(14.50 \%)$, W3C $(2.10 \%)$, and W5C $(1.66 \%)$, suggesting that compounds measured by these electrodes make smaller contributions to differences in flavor between samples.

We used PCA to visualize relationships between samples based on their flavor qualities as detected by the electronic nose (Figure 4). Principal components 1 and 2 accounted for $98.66 \%$ of the total variance $(84.52 \%$ and $14.34 \%$, respectively), suggesting that these first 2 comprehensive variables accounted for most of the original variability. We observed some interesting grouping patterns on the PCA score plot. Samples LFY4, LFY7, LFY9, LFY10, LFY11, and LFY12 formed a cluster at the left side of the plot (Figure 4a), and samples LFY1, LFY2, LFY3, LFY5, LFY6, and LFY8 clustered at the right side. On the loading plot (Figure 4b), most of the flavor parameters clustered to the left, including W1C, W3C, W6S, W5C, W1S, W2S, and W3S, suggesting associations between these parameters and the samples

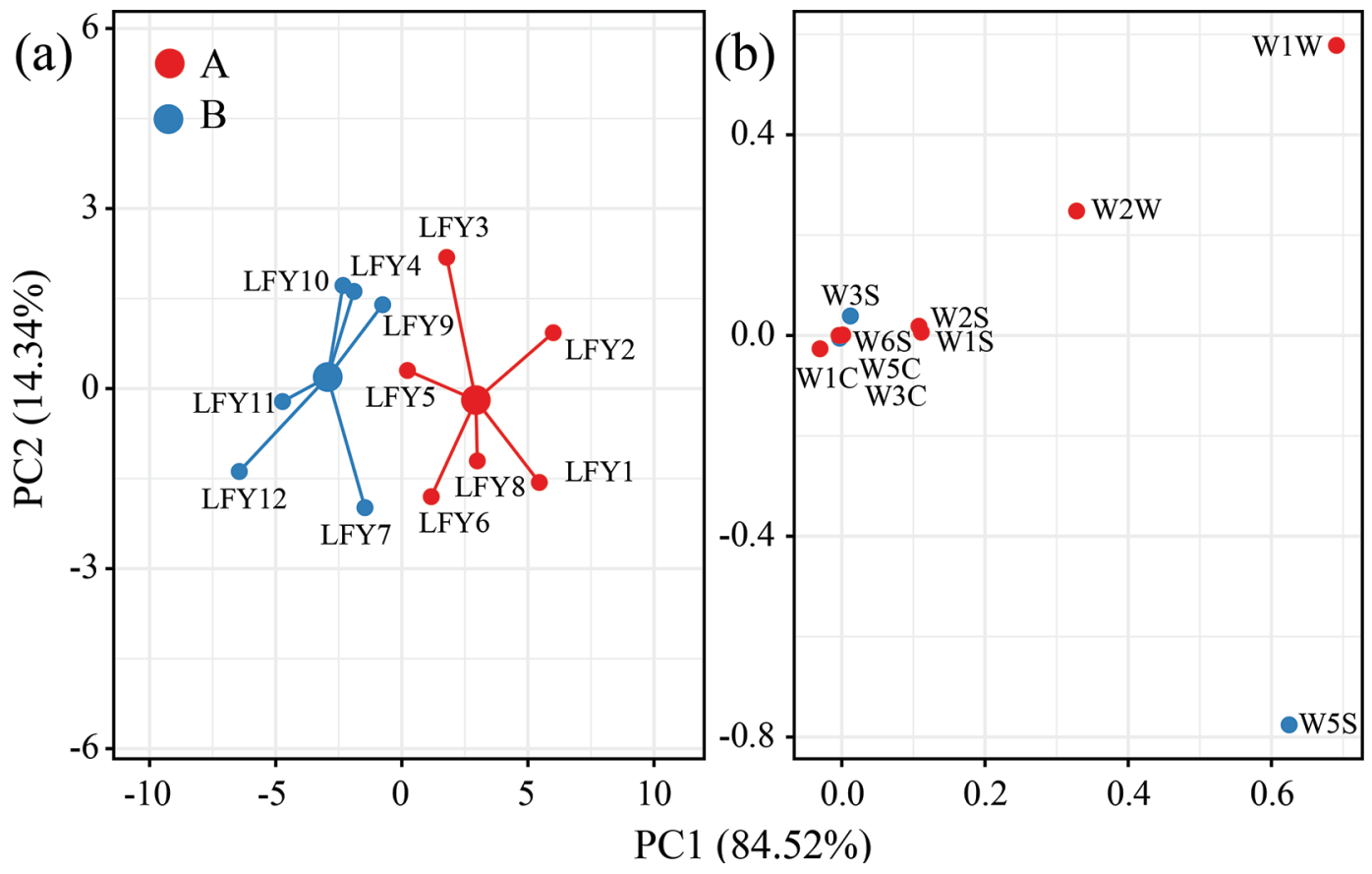

Figure 4. Principal component (PC) analysis (a) score plot, and (b) loading plot for flavor quality in cheese samples from different regions. LFY indicates sample numbers; $\mathrm{W}$ indicates sensor type. 


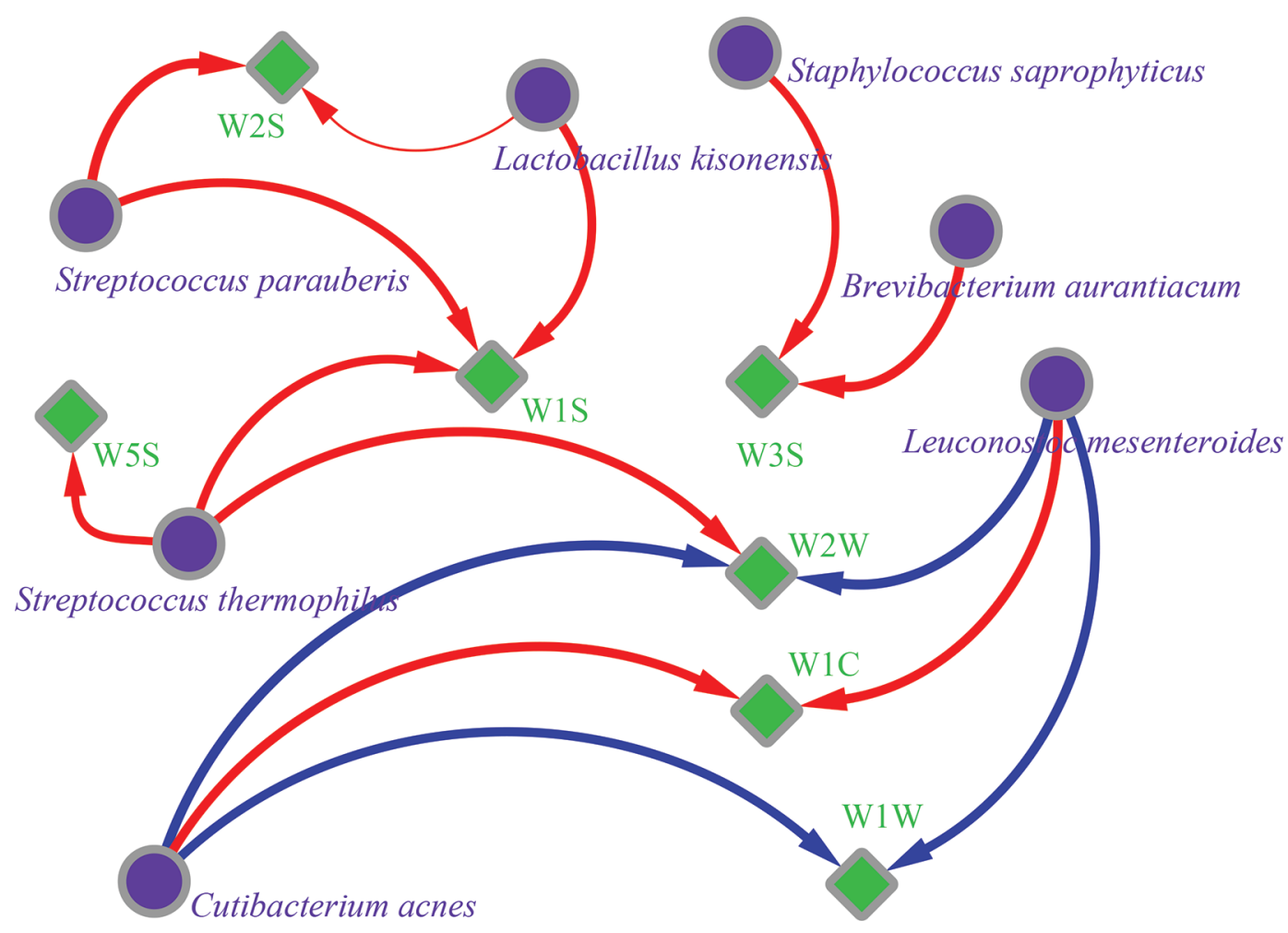

Figure 5. Correlation network diagram of major bacterial species and flavor properties of cheese samples. Purple circles represent dominant bacterial species, and green squares represent flavor indices; red and blue lines represent positive and negative correlations, respectively. Line thickness represents the magnitude of the correlation; W indicates sensor types.

that also clustered at the left side of the score plot. In contrast, samples that clustered at the right side of the score plot showed a stronger association with W2W.

We constructed a correlation network diagram to illustrate the relationship between the dominant cheese bacteria and flavor quality (Figure 5). We included 16 dominant bacterial species (relative abundance $>0.5 \%$ ) in the analysis. We found significant correlations between 7 of the bacteria and 7 flavor indices $(P<0.05$; $\mathrm{r}$ $\leq-0.4$ or $\mathrm{r} \geq 0.4$ ). We found significant positive correlations $(P<0.05)$ for Staphylococcus saprophyticus and Brevibacterium aurantiacum with W3S; for Lactobacillus kisonensis and Streptococcus parauberis with W1S and W2S; and for Strep. thermophilus with W1S, W5S, and $\mathrm{W} 2 \mathrm{~W}$. We found significant negative correlations for Leuconostoc mesenteroides and Cutibacterium acnes with W1W and W2W.

The flavor quality of fermented dairy products is closely related to the bacterial community present (Gemechu, 2015). Some studies have found that Strep. parauberis can produce enzymes needed in the synthesis of linear alkanes and alcohols (Marliere et al., 2016); Staph. saprophyticus and B. aurantiacum can be used to produce some biological active alkanes (Shivarama Holla et al., 2001). The content of olefins and alcohols in chili sauce fermented by Lactobacillus was also significantly high (Lee et al., 2001), and co-fermentation with Leu. mesenteroides and Lc. lactis can make the aroma of dairy products more intense (Bellengier et al., 1997). Our results were consistent with previous reports that these bacteria were correlated with some of the flavor properties of cheese, especially those associated with alkanes, ethanol, sulfides, and aromatics. These findings suggest that the dominant species are key contributors to cheese flavor and are responsible for its unique taste.

Relevant reports show that alkanes in cheese seem to be responsible for its peculiar smell, and nitrogen and oxygen compounds have negative effects on cheese flavor quality. At the same time, the ripening process of cheese is the continuous synthesis of aromatic substances. Ripening can be monitored by measuring the relative strength of aromatic substances and ethanol. Therefore, proper control of Strep. parauberis, Lb. kisonensis, Staph. saprophyticus, B. aurantiacum, and Strep. thermophilus content during the fermentation process is beneficial for controlling the growth of undesirable odors such as alkanes in cheese and improving its flavor. However, in the industrial production of cheese, the appropriate addition of auxiliary fermentation strains 
such as Leu. mesenteroides has shown positive effects on accelerating cheese ripening, shortening production cycles, and improving flavor quality.

\section{Functional Metagenomic Prediction by PICRUSt}

To further understand the potential functional role of the bacterial community in fermentation and flavor formation, we used functional metagenome prediction and enrichment analysis with PICRUSt (Douglas et al., 2018) software and annotated this from the KEGG database (Kanehisa et al., 2017).

Based on PCA, samples were classified into group A (LFY1, LFY2, LFY3, LFY5, LFY6, and LFY8) and group B (LFY4, LFY7, LFY9, LFY10, LFY11, and LFY12). We compared the predicted metagenomes of the 2 sample groups using the Wilcoxon test and we identified 8 significant differential metabolic pathways ( $\mathrm{Z}>1.6$; probability $>80 \%$ or $<20 \%$; Figure $6 \mathrm{a}$ ). All predicted differential metabolic pathways were involved in metabolism in the primary functional layer. Among the 8 metabolic pathways, benzoate degradation had the greatest relative abundance in all cheese samples, followed by styrene degradation, secondary bile acid biosynthesis, and nitrotoluene degradation $(P<0.05)$; we found no significant difference in the relative abundance of other differential metabolic pathways (Figure $6 a, b)$.

The results of the redundancy analysis showed that the metabolic pathways for benzoate degradation, xylene degradation, toluene degradation, and caprolactam degradation were well aligned with the assignment of samples on the constraint axis of the redundancy analysis plot (Figure 6c). The benzoate degradation pathway was located at the left side of the diagram, and the other differential metabolic pathways were located on the right side, indicating that benzoate degradation contributed more to group A cheese samples. Xylene degradation, toluene degradation, and caprolactam degradation contributed more to group B cheese samples.

Benzoate has bacteriostatic activity and is often used in food preservatives. It can inhibit the growth of related bacteria and prevent the production of a bad smell. Xylene and toluene have an aromatic smell, which is beneficial for improving product quality.

We further analyzed the relationship between bacterial communities and metabolic pathways (Figure 7) and found significant positive correlations $(P<0.05)$ for Staph. saprophyticus and $B$. aurantiacum with chlorocyclohexane and the chlorobenzene degradation; and for Lb. kisonensis and Strep. parauberis with 3 metabolic pathways (toluene degradation, caprolactam degradation, and nitrotoluene degradation). In con-
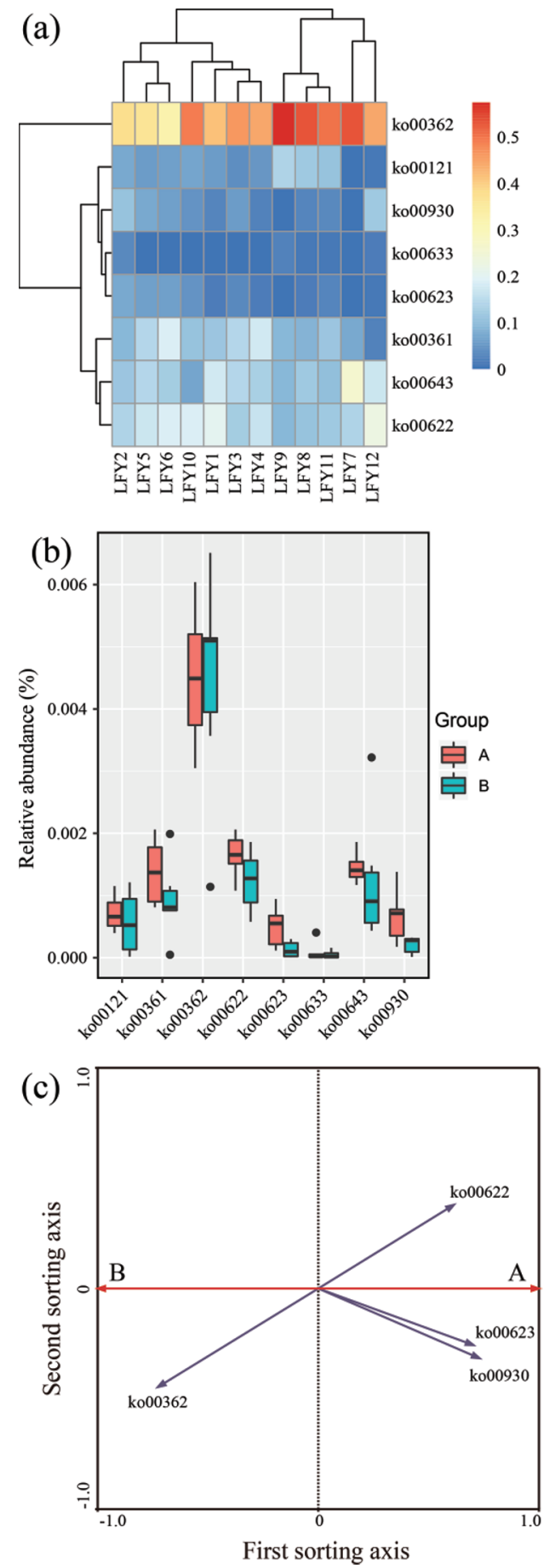

Figure 6. Functional prediction of cheese microbiota. (a) Heat map, and (b) boxplot showing relative abundance of 8 differential metabolic pathways from each sample (box represents the middle $50 \%$ of the entire set of data; line represents the median of the entire set of data; whiskers represent the lower $25 \%$ and upper $25 \%$ of the range of data values, excluding outliers; circles represent outliers); (c) redundancy analysis identifying the key discriminatory pathways. Pathways: ko00121 = secondary bile acid biosynthesis; ko00361 $=$ chlorocyclohexane and chlorobenzene degradation; ko00362 = benzoate degradation; ko00622 = xylene degradation; ko00623 = toluene degradation; ko00633 = nitrotoluene degradation; ko00643 = styrene degradation; ko00930 $=$ caprolactam degradation. LFY indicates sample numbers. 


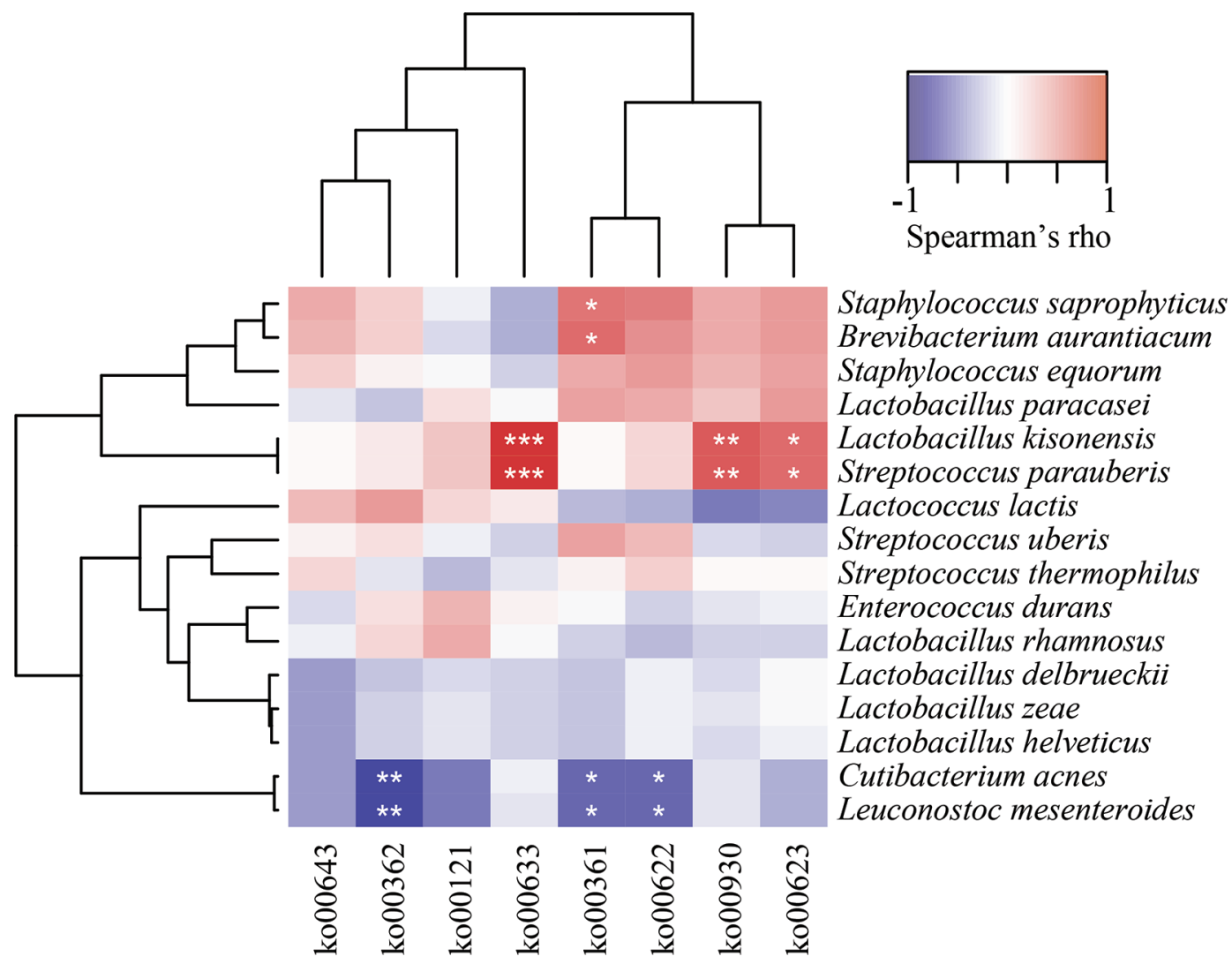

Figure 7. Spearman rank correlation between metabolic pathways and bacterial species. Significant correlations are represented by $* * * P$ $<0.001$, ${ }^{* *} P<0.01$, and ${ }^{*} P<0.05$. Pathways: ko00121 = secondary bile acid biosynthesis; ko00361 = chlorocyclohexane and chlorobenzene degradation; ko00362 = benzoate degradation; ko00622 = xylene degradation; ko00623 = toluene degradation; ko00633 = nitrotoluene degradation; ko00643 = styrene degradation; ko00930 = caprolactam degradation.

trast, we found significant negative correlations for $C$. acnes and Leu. mesenteroides with xylene degradation, chlorocyclohexane and chlorobenzene degradation, and benzoate degradation.

Results from cluster analysis of the different metabolic pathways were similar to the results of PCA (Figure 6a). The cheese samples were divided into 2 clusters, suggesting that the groups of cheese samples had different functional metagenome profiles, which could explain the distinctive flavor characteristics of the 2 groups. The differential expression of pathways relating to xenobiotic biodegradation and metabolism and lipid metabolism could suggest that these pathways play an important role in the synthesis of flavor volatile compounds. Different LAB can give cheeses special flavor characteristics during the metabolism of milk components.

\section{CONCLUSIONS}

This study applied the PacBio SMRT sequencing technology to describe the bacterial communities from cheese collected from different regions. We identified 144 bacterial genera (e.g., Lactobacillus, Streptococcus, Lactococcus, and Staphylococcus) and 217 species (e.g., Lactococcus lactis, Streptococcus thermophilus, Staphylococcus equorum, and Streptococcus uberis). We found large variation among samples at the OTU level. Based on flavor quality as detected by an electronic nose system, the samples clustered into 2 separate groups on the PCA plot. We identified different bacterial species and metabolic pathways, and these discriminating components may be responsible for the distinctive flavor qualities of the cheese samples.

\section{ACKNOWLEDGMENTS}

This research was supported by the National Natural Science Foundation of China (grant number 31622043), the China Agriculture Research System (grant numbers CARS-36), and the Inner Mongolia Science and Technology Projects (grant numbers 201802063), and the program was funded by the University for Fostering Distinguished Young Scholars (grant numbers 
2017XJQ-2). The authors have not stated any conflicts of interest.

\section{REFERENCES}

Aldrete-Tapia, A., M. C. Escobar-Ramirez, M. L. Tamplin, and M. Hernandez-Iturriaga. 2014. High-throughput sequencing of microbial communities in Poro cheese, an artisanal Mexican cheese. Food Microbiol. 44:136-141. https://doi.org/10.1016/j.fm.2014.05 .022 .

Bellengier, P., J. Richard, and C. Foucaud. 1997. Associative growth of Lactococcus lactis and Leuconostoc mesenteroides strains in milk. J. Dairy Sci. 80:1520-1527. https://doi.org/10.3168/jds.S0022 -0302(97)76081-8.

Blaiotta, G., N. Murru, A. Di Cerbo, M. Succi, R. Coppola, and M. Aponte. 2017. Commercially standardized process for probiotic "Italico" cheese production. Lebensm. Wiss. Technol. 79:601-608. https://doi.org/10.1016/j.lwt.2016.11.008.

Bourdichon, F., S. Casaregola, C. Farrokh, J. C. Frisvad, M. L. Gerds, W. P. Hammes, J. Harnett, G. Huys, S. Laulund, A. Ouwehand, I. B. Powell, J. B. Prajapati, Y. Seto, E. Ter Schure, A. Van Boven, V. Vankerckhoven, A. Zgoda, S. Tuijtelaars, and E. B. Hansen. 2012. Food fermentations: Microorganisms with technological beneficial use. Int. J. Food Microbiol. 154:87-97. https://doi.org/10 .1016/j.ijfoodmicro.2011.12.030

Caggianiello, G., M. Kleerebezem, and G. Spano. 2016. Exopolysaccharides produced by lactic acid bacteria: From health-promoting benefits to stress tolerance mechanisms. Appl. Microbiol. Biotechnol. 100:3877-3886. https://doi.org/10.1007/s00253-016-7471-2.

Cao, J., J. Yang, Q. Hou, H. Xu, Y. Zheng, H. Zhang, and L. Zhang. 2017. Assessment of bacterial profiles in aged, home-made Sichuan paocai brine with varying titratable acidity by PacBiol. SMRT sequencing technology. Food Control 78:14-23. https://doi.org/10 .1016/j.foodcont.2017.02.006.

Caporaso, J. G., K. Bittinger, F. D. Bushman, T. Z. DeSantis, G. L. Andersen, and R. Knight. 2010. PyNAST: S flexible tool for aligning sequences to a template alignment. Bioinformatics 26:266-267. https://doi.org/10.1093/bioinformatics/btp636.

Caporaso, J. G., C. L. Lauber, W. A. Walters, D. Berg-Lyons, J. Huntley, N. Fierer, S. M. Owens, J. Betley, L. Fraser, M. Bauer, N. Gormley, J. A. Gilbert, G. Smith, and R. Knight. 2012. Ultrahigh-throughput microbial community analysis on the Illumina HiSeq and MiSeq platforms. ISME J. 6:1621-1624. https://doi .org/10.1038/ismej.2012.8.

Conway, J. R., A. Lex, and N. Gehlenborg. 2017. UpSetR: An R package for the visualization of intersecting sets and their properties. Bioinformatics 33:2938-2940. https://doi.org/10.1093/ bioinformatics/btx364.

Dan, T., D. Wang, S. Wu, R. Jin, W. Ren, and T. Sun. 2017. Profiles of volatile flavor compounds in milk fermented with different proportional combinations of Lactobacillus delbrueckii ssp. bulgaricus and Streptococcus thermophilus. Molecules 22:E1633. https://doi .org $/ 10.3390 /$ molecules22101633.

DeSantis, T. Z., P. Hugenholtz, N. Larsen, M. Rojas, E. L. Brodie, K. Keller, T. Huber, D. Dalevi, P. Hu, and G. L. Andersen. 2006. Greengenes, a chimera-checked 16S rRNA gene database and workbench compatible with ARB. Appl. Environ. Microbiol. 72:5069-5072. https://doi.org/10.1128/AEM.03006-05.

Douglas, G. M., R. G. Beiko, and M. G. I. Langille. 2018. Predicting the functional potential of the microbiome from marker genes using PICRUSt. Methods Mol. Biol. 1849:169-177.

Dugat-Bony, E., L. Garnier, J. Denonfoux, S. Ferreira, A. S. Sarthou, P. Bonnarme, and F. Irlinger. 2016. Highlighting the microbial diversity of 12 French cheese varieties. Int. J. Food Microbiol. 238:265-273. https://doi.org/10.1016/j.ijfoodmicro.2016.09.026.

Edgar, R. C. 2010. Search and clustering orders of magnitude faster than BLAST. Bioinformatics 26:2460-2461. https://doi.org/10 .1093 /bioinformatics/btq461.

Felicio, T. L., E. A. Esmerino, V. A. S. Vidal, L. P. Cappato, R. K. A. Garcia, R. N. Cavalcanti, M. Q. Freitas, C. A. Conte Junior, M.
C. Padilha, M. C. Silva, R. S. L. Raices, D. B. Arellano, H. M. A. Bollini, M. A. R. Pollonio, and A. G. Cruz. 2016. Physico-chemical changes during storage and sensory acceptance of low sodium probiotic Minas cheese added with arginine. Food Chem. 196:628-637. https://doi.org/10.1016/j.foodchem.2015.09.102.

Fujita, A., D. Sarkar, M. I. Genovese, and K. Shetty. 2017. Improving anti-hyperglycemic and anti-hypertensive properties of camu-camu (Myriciaria dubia Mc. Vaugh) using lactic acid bacterial fermentation. Process Biochem. 59:133-140. https://doi.org/10.1016/j .procbio.2017.05.017.

Gemechu, T. 2015. Review on lactic acid bacteria function in milk fermentation and preservation. Afr. J. Food Sci. 9:170-175. https: //doi.org/10.5897/AJFS2015.1276.

Georgalaki, M., G. Zoumpopoulou, E. Mavrogonatou, G. Van Driessche, V. Alexandraki, R. Anastasiou, M. Papadelli, M. Kazou, E. Manolopoulou, D. Kletsas, B. Devreese, K. Papadimitriou, and E. Tsakalidou. 2017. Evaluation of the antihypertensive angiotensinconverting enzyme inhibitory (ACE-I) activity and otherprobiotic properties of lactic acid bacteria isolated from traditional Greek dairy products. Int. Dairy J. 75:10-21. https://doi.org/10.1016/j .idairyj.2017.07.003.

Gilliam, F. S., and N. E. Saunders. 2003. Making more sense of the order: A review of Canoco for Windows 4.5, PC-ORD version 4 and SYN-TAX 2000. J. Veg. Sci. 14:297-304. https://doi.org/10 .1111/j.1654-1103.2003.tb02155.x.

Hai, Z., and J. Wang. 2006. Electronic nose and data analysis for detection of maize oil adulteration in sesame oil. Sens. Actuators B Chem. 119:449-455. https://doi.org/10.1016/j.snb.2006.01.001.

Hammer, Ø., D. A. T. Harper, and P. D. Ryan. 2001. PAST: Paleontological statistics software package for education and data analysis. Palaeontol. Electronica 4:1-9.

Holla, B. S., R. Gonsalves, B. Sooryanarayana Rao, S. Shenoy, and H. N. Gopalakrishna. 2001. Synthesis of some new biologically active bis-(thiadiazolotriazines) and bis-(thiadiazolotriazinyl) alkanes. Farmaco 56:899-903. https://doi.org/10.1016/S0014 $-827 \mathrm{X}(01) 01119-3$.

Hou, Q., H. Xu, Y. Zheng, X. Xi, L.-Y. Kwok, Z. Sun, H. Zhang, and W. Zhang. 2015. Evaluation of bacterial contamination in raw milk, ultra-high temperature milk and infant formula using single molecule, real-time sequencing technology. J. Dairy Sci. 98:84648472. https://doi.org/10.3168/jds.2015-9886.

Jin, H., L. Mo, L. Pan, Q. Hou, C. Li, I. Darima, and J. Yu. 2018. Using PacBio sequencing to investigate the bacterial microbiota of traditional Buryatian cottage cheese and comparison with Italian and Kazakhstan artisanal cheeses. J. Dairy Sci. 101:6885-6896. https://doi.org/10.3168/jds.2018-14403.

Yeluri Jonnala, B. R., P. L. H. McSweeney, J. J. Sheehan, and P. D. Cotter. 2018. Sequencing of the cheese microbiome and its relevance to industry. Front. Microbiol. 9:1020.

Kamimura, B. A., F. De Filippis, A. S. Sant'Ana, and D. Ercolini. 2019. Large-scale mapping of microbial diversity in artisanal Brazilian cheeses. Food Microbiol. 80:40-49. https://doi.org/10.1016/ j.fm.2018.12.014.

Kanehisa, M., M. Furumichi, M. Tanabe, Y. Sato, and K. Morishima. 2017. KEGG: New perspectives on genomes, pathways, diseases and drugs. Nucleic Acids Res. 45(D1):D353-D361. https://doi .org/10.1093/nar/gkw1092.

Koca, N., Z. Erbay, and F. Kaymak-Ertekin. 2015. Effects of spraydrying conditions on the chemical, physical, and sensory properties of cheese powder. J. Dairy Sci. 98:2934-2943. https://doi.org/10 $.3168 /$ jds.2014-9111.

Koren, S., M. C. Schatz, B. P. Walenz, J. Martin, J. T. Howard, G. Ganapathy, Z. Wang, D. A. Rasko, W. R. McCombie, E. D. Jarvis, and A. M. Phillippy. 2012. Hybrid error correction and de novo assembly of single-molecule sequencing reads. Nat. Biotechnol. 30:693-700. https://doi.org/10.1038/nbt.2280.

Lee, S. M., J. Y. Lee, Y. J. Cho, M. S. Kim, and Y. S. Kim. 2018. Determination of volatiles and carotenoid degradation compounds in red pepper fermented by Lactobacillus parabuchneri. J. Food Sci. 83:2083-2091. https://doi.org/10.1111/1750-3841.14221. 
Licitra, G., M. Caccamo, F. Valence, S. Lortal. 2018. Traditional wooden equipment used for cheesemaking and their effect on quality. Pages 157-172 in Global Cheesemaking Technology: Cheese Quality and Characteristics. P. Papademas and T. Bintsis, ed. Wiley, Hoboken, NJ.

Lima-Ojeda, J. M., R. Rupprecht, and T. C. Baghai. 2017. "I am I and my bacterial circumstances": Linking gut microbiome, neurodevelopment, and depression. Front. Psychiatry 8:153. https://doi.org/ 10.3389/fpsyt.2017.00153.

Linares, D. M., C. Gómez, E. Renes, J. M. Fresno, M. E. Tornadijo, R. P. Ross, and C. Stanton. 2017. Lactic acid bacteria and Bifidobacteria with potential to design natural biofunctional healthpromoting dairy foods. Front. Microbiol. 8:846. https://doi.org/10 $.3389 /$ fmicb.2017.00846.

Liu, X., and A. Narbad. 2018. Lactic acid bacteria-based food fermentations. Pages 141-181 in Lactic Acid Bacteria in Foodborne Hazards Reduction. Springer, Singapore.

Maidak, B. L., J. R. Cole, T. G. Lilburn, C. T. Parker Jr., P. R. Saxman, J. M. Stredwick, G. M. Garrity, B. Li, G. J. Olsen, S. Pramanik, T. M. Schmidt, and J. M. Tiedje. 2000. The RDP (ribosomal database project) continues. Nucleic Acids Res. 28:173-174. https://doi.org/10.1093/nar/28.1.173.

Mania, I., A. M. Delgado, C. Barone, and S. Parisi. 2018. The ExTra Tool-practical simulations in the cheesemaking industry when using cheeses, butter and rennet caseins. Pages 67-93 in Traceability in the Dairy Industry in Europe. Springer Nature, Basel, Switzerland.

Marliere, P., M. Delcourt, M. Anissimova, and R. Tallon. 2016. Production of alkenes by combined enzymatic conversion of 3-hydroxyalkanoic acids. Assignee Global Bioenergies. US Patent Application $14 / 886,437$.

O'Sullivan, D. J., P. D. Cotter, O. O'Sullivan, L. Giblin, P. L. H. McSweeney, and J. J. Sheehan. 2015. Temporal and spatial differences in microbial composition during the manufacture of a continentaltype cheese. Appl. Environ. Microbiol. 81:2525-2533. https://doi .org/10.1128/AEM.04054-14.

Pedersen, T. B., F. K. Vogensen, and Y. Ardo. 2016. Effect of heterofermentative lactic acid bacteria of DL-starters in initial ripening of semi-hard cheese. Int. Dairy J. 57:72-79. https://doi.org/10 .1016/j.idairyj.2016.02.041.

Pogačić, T., M.-B. Maillard, A. Leclerc, C. Hervé, V. Chuat, F. Valence, and A. Thierry. 2016. Lactobacillus and Leuconostoc volatilomes in cheese conditions. Appl. Microbiol. Biotechnol. 100:23352346. https://doi.org/10.1007/s00253-015-7227-4.

Quast, C., E. Pruesse, P. Yilmaz, J. Gerken, T. Schweer, P. Yarza, J. Peplies, and F. O. Glöckner. 2013. The SILVA ribosomal RNA gene database project: Improved data processing and web-based tools. Nucleic Acids Res. 41(D1):D590-D596. https://doi.org/10 $.1093 / \mathrm{nar} / \mathrm{gks} 1219$.

Rhoads, A., and K. F. Au. 2015. PacBiol. sequencing and its applications. Genomics Proteomics Bioinformatics 13:278-289. https:// doi.org/10.1016/j.gpb.2015.08.002.

Sádecká, J., N. Šaková, D. Pangallo, J. Koreňová, E. Kolek, A. Puškárová, M. Bucková, L. Valík, and T. Kuchta. 2016. Microbial diversity and volatile odour-active compounds of barrelled ewes' cheese as an intermediate product that determines the quality of winter bryndza cheese. Lebensm. Wiss. Technol. 70:237-244. https://doi.org/10.1016/j.lwt.2016.02.048.
Salameh, C., S. Banon, C. Hosri, and J. Scher. 2016. An overview of recent studies on the main traditional fermented milks and white cheeses in the Mediterranean region. Food Rev. Int. 32:256-279. https://doi.org/10.1080/87559129.2015.1075210.

Samelis, J., and A. Kakouri. 2019. Major technological differences between an industrial-type and five artisan-type Greek PDO Galotyri market cheeses as revealed by great variations in their lactic acid microbiota. AIMS Agric. Food 4:685-710. https://doi.org/10 .3934/agrfood.2019.3.685.

Scatassa, M. L., R. Gaglio, G. Macaluso, N. Francesca, W. Randazzo, C. Cardamone, A. Di Grigoli, G. Moschetti, and L. Settanni. 2015. Transfer, composition and technological characterization of the lactic acid bacterial populations of the wooden vats used to produce traditional stretched cheeses. Food Microbiol. 52:31-41. https://doi.org/10.1016/j.fm.2015.06.008.

Schloss, P. D., M. L. Jenior, C. C. Koumpouras, S. L. Westcott, and S. K. Highlander. 2016. Sequencing 16S rRNA gene fragments using the PacBiol. SMRT DNA sequencing system. PeerJ 4:e1869.

Sørensen, K. I., M. Curic-Bawden, M. P. Junge, T. Janzen, and E. Johansen. 2016. Enhancing the sweetness of yoghurt through metabolic remodeling of carbohydrate metabolism in Streptococcus thermophilus and Lactobacillus delbrueckii ssp. bulgaricus. Appl. Environ. Microbiol. 82:3683-3692. https://doi.org/10.1128/AEM $.00462-16$.

Stellato, G., F. De Filippis, A. La Storia, and D. Ercolini. 2015. Coexistence of lactic acid bacteria and potential spoilage microbiota in a dairy processing environment. Appl. Environ. Microbiol. 81:7893-7904. https://doi.org/10.1128/AEM.02294-15.

Stone, W., O. Kroukamp, D. R. Korber, J. McKelvie, and G. M. Wolfaardt. 2016. Microbes at surface-air interfaces: The metabolic harnessing of relative humidity, surface hygroscopicity, and oligotrophy for resilience. Front. Microbiol. 7:1563. https://doi.org/10 .3389 /fmicb.2016.01563.

Treu, L., B. de Diego-Díaz, K. Papadimitriou, E. Tsakalidou, A. Giacomini, and V. Corich. 2017. Whole-genome sequences of three Streptococcus macedonicus strains isolated from Italian cheeses in the Veneto region. Genome Announc. 5:e01358-17. https://doi .org/10.1128/genomeA.01358-17.

Wagner, J., P. Coupland, H. P. Browne, T. D. Lawley, S. C. Francis, and J. Parkhill. 2016. Evaluation of PacBiol. sequencing for fulllength bacterial 16S rRNA gene classification. BMC Microbiol. $16: 274$.

Wang, J., Y. Zheng, X. Xi, Q. Hou, H. Xu, J. Zhao, J. Li, Y. Bian, H. Ma, Y. Wang, L. Y. Kwok, H. Zhang, and Z. Sun. 2018. Application of PacBiol. Single Molecule Real-Time (SMRT) sequencing in bacterial source tracking analysis during milk powder production. Food Control 93:226-234. https://doi.org/10.1016/j.foodcont.2018 .05 .044 .

Zeidan, A. A., V. K. Poulsen, T. Janzen, P. Buldo, P. M. F. Derkx, G. Oregaard, and A. R. Neves. 2017. Polysaccharide production by lactic acid bacteria: rom genes to industrial applications. FEMS Microbiol. Rev. 41(Supp_1):S168-S200. https://doi.org/10.1093/ femsre/fux017.

Zheng, X., F. Liu, K. Li, X. Shi, Y. Ni, B. Li, and B. Zhuge. 2018 Evaluating the microbial ecology and metabolite profile in Kazak artisanal cheeses from Xinjiang, China. Food Res. Int. 111:130136. https://doi.org/10.1016/j.foodres.2018.05.019. 